\title{
Atomic-Scale Aspects of Oriented Attachment
}

\author{
Kristen A. Fichthorn \\ Department of Chemical Engineering and Department of Physics \\ The Pennsylvania Sate University, University Park, PA 16802
}

\begin{abstract}
Oriented attachment (OA), or the non-random aggregation of crystallites to form single or twinned crystals, has been observed is a variety of different systems during crystal growth. OA is believed to underlie the growth of anisotropic and potentially useful nanostructures, such as wires and plates, as well as complex hierarchical nanostructures. However, its origins are poorly understood. I review insights into OA that we gained in two sets of molecular-dynamics simulation studies of titanium dioxide (anatase) nanocrystals. In the first set of studies, we focused on the role of intrinsic nanocrystal forces in facilitating nanocrystal alignment and aggregation in vacuum. These studies show that, although nanocrystal aggregation occurs in a predictable way, OA is not a common outcome. In a second set of studies, we used the ReaxFF reactive force field to study anatase nanocrystal aggregation in an aqueous (humid) environment. OA occurs in these studies and is mediated by adsorbed water and surface hydroxyls. The OA mechanisms that we find for anatase may be common to other aqueous metal-oxide systems.

Keywords: oriented attachment; crystal growth; nanoscience; titanium dioxide; molecular dynamics
\end{abstract}

Email address: fichthorn@psu.edu (Kristen A. Fichthorn)

Preprint submitted to Chemical Engineering Science

April 30, 2014 


\section{Introduction}

Solution-phase syntheses have been extensively studied and employed to fabricate solid nanocrystals with well-defined sizes and shapes. Studies of the growth of these nanocrystals are both interesting and beneficial. From a fun-

5 damental standpoint, many crystals in solution grow via Ostwald ripening, in which the larger nanocrystals grow, atom-by-atom, at the expense of smaller crystallites [1]. An alternate or parallel mechanism is cluster aggregation, which often leads to the formation of random and ill-formed agglomerates. However, cluster aggregation is not always random. As Penn and Banfield demonstrated in 1999, cluster aggregation can occur along specific crystallographic directions and lead to the formation of single or twinned crystallites [2]. This non-random aggregation is known as oriented attachment $(\mathrm{OA})$.

Since the initial discovery of OA, there have been numerous studies of this fascinating phenomenon $[3,4,5]$. From a practical perspective, OA frequently underlies the growth of complex architectures ranging from quasi-one and twodimensional structures $[6,7,8,9,10,11,12,13,14,15]$, such as nanowires, nanoribbons, and nanosheets, to hierarchical two- or three- dimensional superstructures $[16,17,18]$. In the interest of achieving controlled bottom-up (and often surfactant-free) synthesis of these potentially useful structures, a strong fundamental understanding of OA is highly beneficial.

How does OA come about? Many studies cite the reduction of interfacial energy as the driving force for OA. For example, if the aggregation of two nanocrystals occurs on the facets with the highest surface energies, then these interfaces are eliminated and the aggregate has a reduced interfacial energy. 25 Although this may be an appropriate driving force in many systems, it does not explain, mechanistically, what makes two nanocrystals move toward each other and choose a specific orientation (from the very large set of all possible orientations) that causes them to join in perfect registry. Very recent experiments [19] with high-resolution transmission electron microscopy (HRTEM) in so a liquid cell have allowed for in situ imaging of OA and can provide insight into 
its origins. However, such experiments do not provide chemically and spatially detailed information on species at the interfaces where OA occurs, which are probably vital for a true understanding of its origins.

Atomic-scale investigations could contribute significantly to understanding

35 OA. Although it is currently challenging to simulate the aggregation of nanometersized particles in solvent using first-principles methods, this can be done in classical molecular dynamics (MD) simulations [20, 21, 22, 23, 24, 25, 26, 27]. MD simulations can also be used to resolve interparticle forces [28, 29, 30, 31, 32]. Provided that MD simulations are based on sufficiently realistic force fields, they can potentially furnish many details of OA that are not accessible experimentally.

OA has been observed for various oxide materials $[2,3,4,5,15,19]$. Indeed, some of the most detailed experimental studies to date have been carried out for this class of materials [19], so that complementary theoretical studies will be useful. In the work I will discuss below, we focused on the aggregation of the anatase polymorph of $\mathrm{TiO}_{2}$. OA was first observed in experimental studies of the crystallization of colloidal (hydrothermal) anatase [2]. In a colloidal system, there are several phenomena that could affect the alignment of two approaching nanocrystals, including intrinsic forces between nanoparticles, selective adsorption and surface chemistry of liquid-phase molecules at the nanoparticle-liquid interface, and solvent-mediated interactions. In this paper, I will discuss insights into OA that we gained in two sets of MD-simulation studies. In the first set, we focused on the role of intrinsic nanocrystal forces in facilitating anatase nanocrystal alignment and aggregation, by studying the aggregation of two anatase crystals in vacuum [24]. Although these studies indicate that intrinsic nanocrystal forces can induce specific (and non-random) aggregation of anatase, we do not observe OA in vacuum. In a second set of studies, we probed the influence of water on anatase nanocrystal aggregation [27]. Interestingly, OA does occur in this system and I will discuss its origins. 
(a)

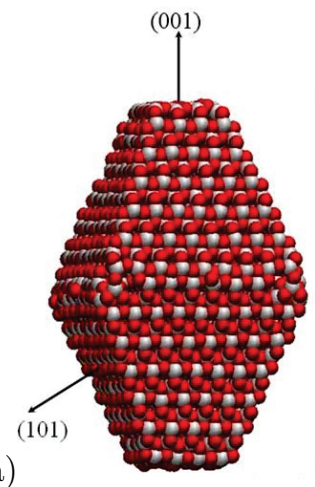

(b)

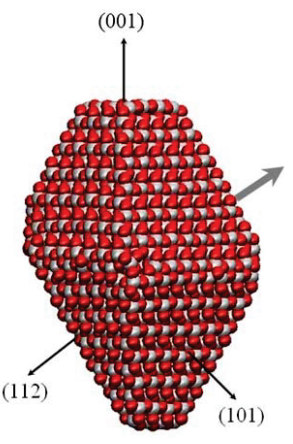

Figure 1: Equilibrated structures of (a) a Wulff-shaped nanocrystal and (b) a nanocrystal that is truncated on the $\{112\}$ facet. Oxygen atoms are shown in red and titanium atoms are shown in white. The arrow in (b) represents the direction of the nanocrystal dipole moment.

To understand the role of intrinsic nanocrystal forces in promoting OA, we consider the aggregation of anatase nanocrystals that possess variations of the experimentally observed [2] and theoretically predicted [33, 34] Wulff shape. As shown in Fig. 1(a), nanocrystals with the Wulff shape contain both $\{101\}$ and $\{001\}$ facets. In addition, we considered 3 possible off-Wulff shapes that could occur during crystal growth [24] and an example containing a $\{112\}$ facet is shown in Fig. 1(b). $\{112\}$ facets have been experimentally observed to play a significant role in the OA of colloidal anatase [2], so by including these we can investigate this possible role. All together, we studied five different nanocrystals: a large (3774 atoms) and a small (816 atoms) Wulff-shaped crystal and three off-Wulff crystals with 2958, 3141, and 3528 atoms. The longest dimensions of these nanocrystals ranged from $6 \mathrm{~nm}$ for the two nanocrystals shown in Fig. 1 to $3 \mathrm{~nm}$ for the small Wulff crystal.

To describe interactions between the nanocrystals, we use the Matsui-Akaogi force field [35]. In this force field, the interaction energy $U$ between atoms $i$ and 
$j$ separated by a distance of $r_{i j}$ has the form

$$
U\left(r_{i j}\right)=A_{i j} \exp \left(-\frac{r_{i j}}{\rho_{i j}}\right)-\frac{C_{i j}}{r_{i j}^{6}}+\frac{q_{i} q_{j}}{r_{i j}} .
$$

The Matsui-Akaogi force field assigns fixed partial charges $q$ of +2.196 and -1.098 to titanium and oxygen, respectively $\left(q_{O}=-q_{T i} / 2\right)$. These effective phonon dispersion of rutile [36]. In addition to the electrostatic term, the first two terms in Eq. (1) constitute a Buckingham potential. The parameters for the Buckingham potential, $A_{i j}, \rho_{i j}$ and $C_{i j}$, are given in ref. 35 .

We justified our use of the Matsui-Akaogi force field in a previous paper [24]. Briefly, in a study of nine promising force fields by Collins and Smith [37], this potential was shown to be the best at reproducing the lattice energy, polymorphic structures, elastic and dielectric constants, and relative surface energies of $\mathrm{TiO}_{2}$. Further, this potential has been useful in a wide variety of applications $[38,39,40,41,42,43,44,45]$. The Matsui-Akaogi force field has demanding variable charge model [46, 47, 48].

We note that electrostatic interactions dominate inter-particle forces in the Matsui-Akaogi force field [24]. Considering the nature and influence of electrostatic interactions on $\mathrm{OA}$, we note that dipole-dipole interactions have been proposed to be the driving force for directed aggregation in some previous studies $[3,4,49,50]$. To assess the possible role of dipole interactions in aggregation, we calculated the dipole moments of all the crystals. The dipole moment $\mu$ is given by $\mu=\sum_{i} q_{i} \overline{r_{i}}$, where $q_{i}$ and $\overline{r_{i}}$ are the charge and position vector of ion $i$, respectively. While nanocrystals with the Wulff shape do not possess a permanent dipole moment due to their symmetry, the off-Wulff nanocrystals that we considered all have permanent dipole moments. For example, the Matsui-Akaogi force field predicts that the nanocrystal shown in Fig. 1(b) has a permanent dipole moment of $\mu=75$ Debye and the direction of its dipole vector is shown in the figure. By creating nanocrystals with different magnitudes and 105 
aligning the nanocrystals prior to their aggregation to see if this alignment is an important mechanism of OA.

As a second way of characterizing inter-particle electrostatic interactions, we made maps of the electrostatic potential surrounding the nanocrystals. To calculate the electrostatic potential, we set up a grid of points in a plane parallel to each nanocrystal facet and summed the electrostatic interactions between a positive charge and all the nanocrystal ions at each grid point. Figure 2 shows the electrostatic maps for a Wulff and an off-Wulff nanocrystal. As we will discuss below, these electrostatic maps were useful in understanding nanocrystal aggregation.
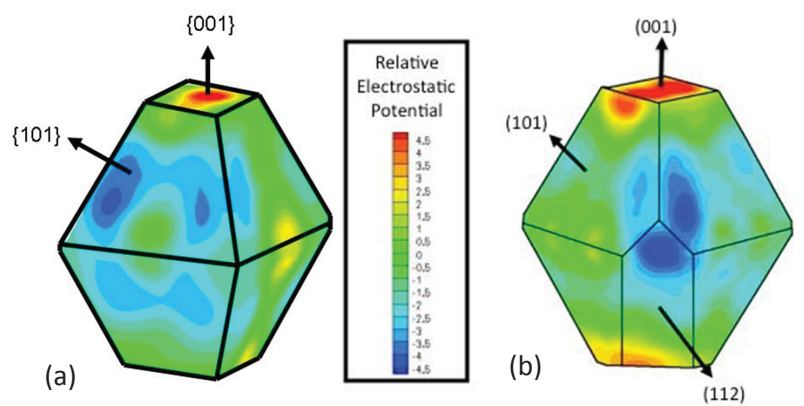

Figure 2: Projected map of the electrostatic potential obtained in planes parallel to and 6 $\AA$ from the facets around (a) a Wulff-shaped nanocrystal and (b) an off-Wulff nanocrystal with a $\{112\}$ facet.

To link these measures with aggregation mechanisms, we used the DL-POLY package [51], version 2.18, to simulate nanocrystal aggregation. Details of these simulations were discussed previously [24]. A nanocrystal was constructed and equilibrated for a time period ranging between $250 \mathrm{ps}$ and $2 \mathrm{~ns}$ in the canonical ensemble at a temperature of $573 \mathrm{~K}$, which is characteristic of hydrothermal syntheses. Subsequent to equilibration, we replicated the nanocrystals and placed them in different initial configurations corresponding to various center-of-mass separations and orientations relative to one another. Initially, the crystals were always sufficiently far apart that they could rotate freely without touching. The 
total simulation times in the two-particle runs ranged between 1.0 and $5.0 \mathrm{~ns}$, which is long enough to observe the approach, coalescence, and initial restructuring of the nanocrystals after coalescence. Actually, we characterized the subsequent restructuring of the nanocrystals in a separate study [26]. Because our anatase aggregates are above the critical size at which rutile becomes more stable than anatase, we observe the anatase-to-rutile transformation in our aggregates and various simulated aspects of transformation are in agreement with experiment [26].

In Fig. 3, we show an aggregation configuration for Wulff-shaped nanocrystals, which is representative of that observed in 47 out of 50 total trajectories for the Wulff-shaped nanocrystals and in 34 out of 40 total trajectories for the off-Wulff nanocrystals. As we see in Fig. 3, aggregation begins when an $\{001\}$ surface of one crystal contacts the edge between two $\{101\}$ surfaces of the other crystal to form a "hinge" that joins the two nanocrystals at/near their edges. Subsequently, one of the particles pivots about the junction, so that the particles are in contact at their facets, and a long-time restructuring process ensues $[26]$.

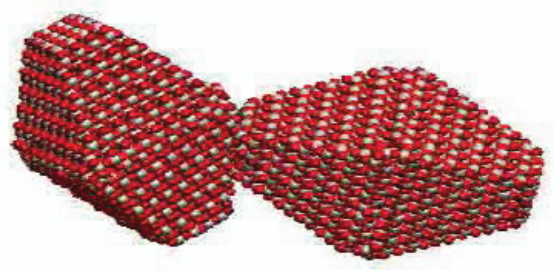

Figure 3: Snapshot of the initial contact of two Wulff-shaped nanocrystals. Oxygen atoms are shown in red and titanium atoms are shown in white.

Regarding the origins of the observed aggregation preference for the Wulff nanocrystals, we note that this cannot be explained by dipole-dipole interactions because the Wulff-shaped nanocrystals have essentially no dipole moment. We verified that the initially zero value of the dipole moment was maintained throughout the simulation until the time of aggregation [24]. In Fig. 2(a), we 
show the electrostatic map surrounding a Wulff-shaped nanocrystal. Here, we see that the electrostatic potential is the most negative around the edges between two $\{101\}$ facets, which can be linked to the high density of undercoordinated O atoms in these areas [24]. Similarly, the electrostatic potential is the most positive on the $\{001\}$ facets, which reflects the presence of under-coordinated $\mathrm{Ti}$ atoms there [24]. Thus, the observed aggregation preference can be attributed to attraction between the negative and positive regions of the electrostatic potential.

Interestingly, the off-Wulff nanocrystals exhibit similar aggregation mechanisms to the Wulff shapes, despite the fact that they possess permanent dipole moments. In all 40 trajectories for the off-Wulff nanocrystals, the dipole moments remained virtually constant throughout the simulation and we only observed one instance of aggregation along the dipole moment. In 26 out of 40 trajectories, the aggregation mechanism for these nanocrystals was identical to that for the Wulff-shaped ones. Following the general principle of local electrostatic attraction that we observed for the Wulff nanocrystals [cf., Fig. 2(a)], an additional aggregation pathway can be attributed to the high density of undercoordinated oxygen atoms on the edges between the $\{112\}$ and $\{101\}$ facets [cf., Fig. 2(b)] in eight out of 40 runs.

Thus, we observe that anatase nanocrystals aggregate with certain preferred orientations in a vacuum environment. Electrostatic forces between undercoordinated atoms on the edges between nanocrystal facets drive this phenomenon. Although dipole-dipole interactions have been inferred to be the driving force for directed aggregation in some previous studies [3, 4, 49, 50], we find that aggregation does not occur preferentially along the direction of the dipole - even when the permanent dipole moment is as large as 250 Debye. Over the nanometer separations at which nanocrystal aggregation occurs, this phenomenon is governed by the local electrostatic environment. 


\section{Oriented Attachment of Nanocrystalline Anatase in an Aqueous Environment}

The absence of OA in our vacuum studies [24] led us to hypothesize that water may play a role in achieving OA. Thus, we considered the aggregation of anatase nanocrystals in an aqueous environment [27]. To properly describe this environment, including the possibility that water can dissociate on the nanocrystal surfaces, we base our MD simulations on the ReaxFF reactive force field. Here we employ the $\mathrm{Ti} / \mathrm{O} / \mathrm{H}$ ReaxFF parameter set that was recently developed by Kim et al. [52]. We performed extensive testing of this force field for various water/TiO2 interfaces [53] and we find that this force field yields good agreement with results from first-principles density functional theory and experiment.

In the experimental studies where OA was first observed, the anatase nanocrystals were in a hydrothermal environment (i.e., in liquid water at a high temperature and pressure) [2]. However, MD simulations of nanocrystals at hydrothermal conditions are prohibitively slow. Thus, our aim in these studies was to achieve an aqueous environment representative of bulk water within the interparticle gap when the nanocrystals are in close proximity. We placed enough water in the simulation box to produce 5 water layers on the particle surfaces at the experimental temperature of $573 \mathrm{~K}$ [2] and we simulated the nanoparticles in the canonical ensemble. In this environment, the nanoparticles are surrounded by water vapor and they can readily approach one another. However, their surfaces are saturated with water and surface hydroxyls. Thus, when they come into close contact, the water confined in the interparticle gap between them is what we would expect to find in bulk water.

We observed numerous instances of $\mathrm{OA}$ in simulations including water and we find that water plays multiple roles in mediating OA. First, adsorbed surface species (molecularly and dissociatively adsorbed water) passivate the nanocrystal surfaces and prevent their immediate aggregation, in contrast to their behavior in vacuum. This allows the nanocrystals to explore many possible different 


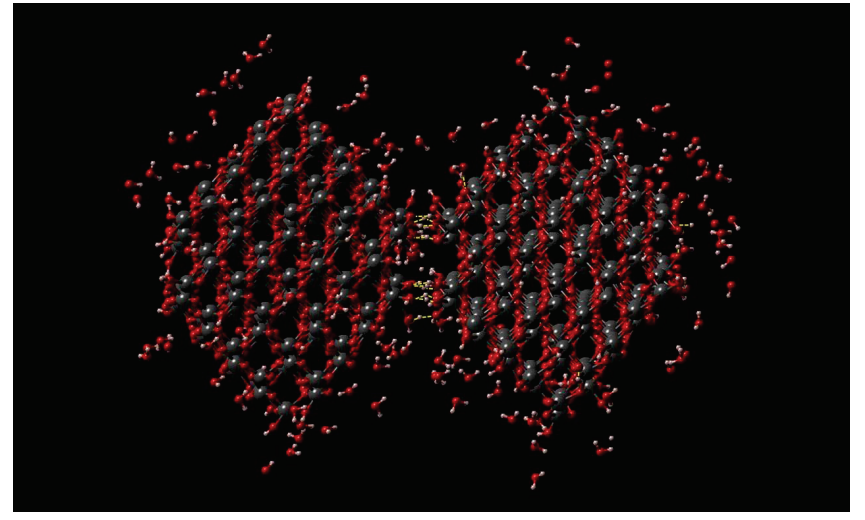

Figure 4: Snapshot of two nanocrystals aligned on their $\{112\}$ facets including the water molecules in their immediate vicinity. Oxygen atoms are shown in red, titanium atoms are shown in gray, hydrogen atoms are shown in white, and hydrogen bonds are shown as dashed, yellow lines.

configurations before they finally aggregate. After the nanocrystals make initial contact, they tend to exist in a loosely associated state, in which they make many transient contacts until they come into close contact, with parallel facets at separations of around $3 \AA$. When the nanoparticles come into close contact, they remain in close contact, held by the crystal field and a network of hydrogen bonds. In this state, they explore numerous relative orientations by "crawling" or sliding their surfaces relative to one another - videos of this process can be viewed in the Supporting Information associated with reference 27. It appears that the nanocrystals probe various possible states until they eventually fall into alignment in a configuration for which their aggregation would lead to OA. The nanoparticles remain in this aligned state for a long time period prior to their eventual coalescence. A snapshot of two nanoparticles aligned along a $\{112\}$ facet is shown in Fig. 4.

To provide a more detailed picture of nanocrystal alignment, we show a close-up view of the interparticle gap between two aligned nanocrystals in Fig. 5. Here, we see the surface species that dominate the interparticle gap: bridging hydroxyls, in which the proton from a dissociated water has bonded to a surface 


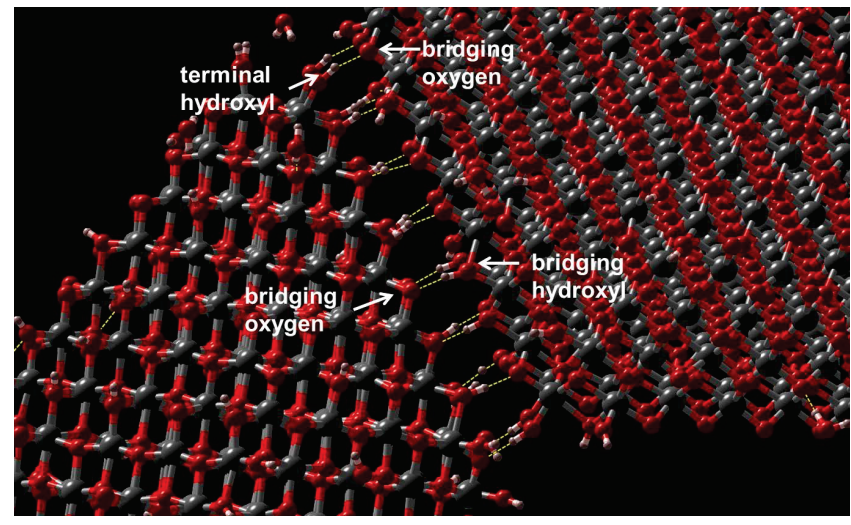

Figure 5: Snapshot of the interparticle gap between two nanocrystals aligned on their $\{112\}$ facets. Oxygen atoms are shown in red, titanium atoms are shown in gray, hydrogen atoms are shown in white, and hydrogen bonds are shown as dashed, yellow lines.

oxygen of the $\mathrm{TiO}_{2}$ nanocrystal; terminal hydroxyls, which form when the $\mathrm{OH}$ group from dissociated water bonds with a Ti surface atom; and bridging oxygens inherent to the $\mathrm{TiO}_{2}$ surface. Hydrogen bonds form between these surface species and these bonds are continually reconfiguring as the nanocrystals orient themselves by diffusing/sliding relative to one another in the associated state.

Nanocrystal aggregation occurs when surface hydroxyls combine with protons and evacuate the interparticle gap as water, leaving bare $\mathrm{TiO}_{2}$ surfaces free to fuse. The time scale over which this reaction occurs is not accessible in our 230 MD simulations at temperatures of $573 \mathrm{~K}$. Thus, we increased the temperature to $1100 \mathrm{~K}$ to accelerate the recombination so we could observe aggregation of the aligned nanocrystals. Aggregation initiates at a contact point and proceeds in a zipper mechanism, closing down the inter-particle gap (a video of this process can be viewed in the Supporting Information of ref. 27). The aggregation mechanism shown in Fig. 6 is representative of the aggregation mechanism observed in water. Surrounding water molecules play an important role in aggregation by mediating proton transfer in the recombination of hydroxyl groups with protons to form water and by stabilizing these ejected water molecules through hydrogen bonding. 


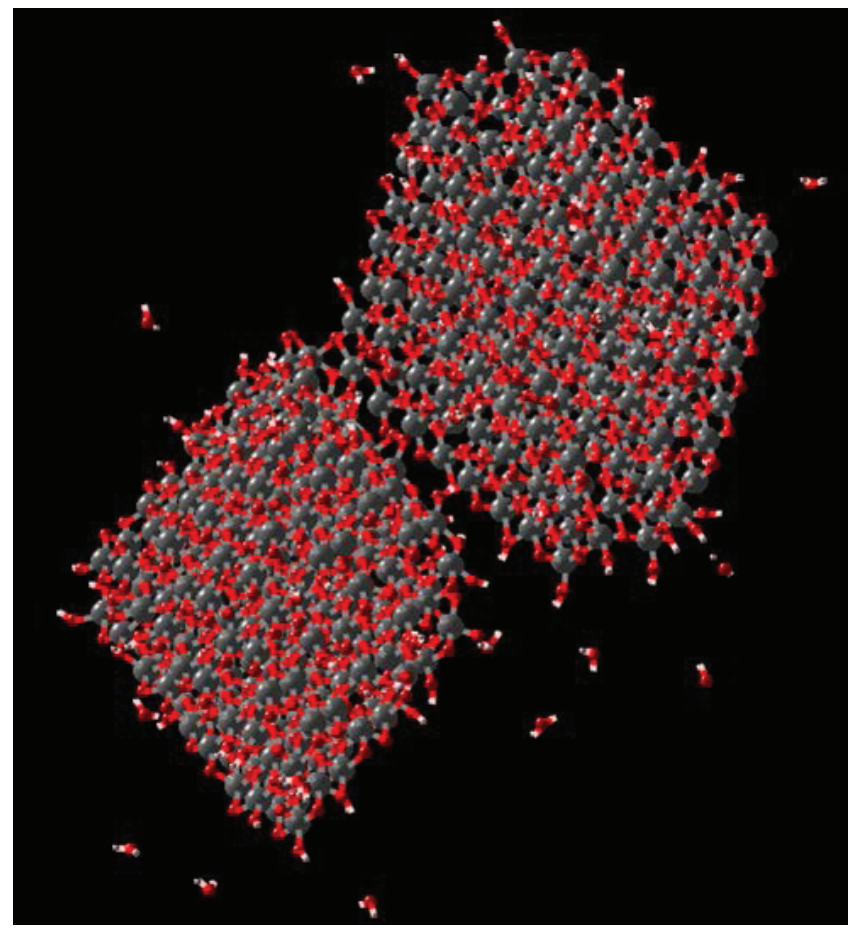

Figure 6: Snapshot of two nanocrystals joining in OA on $\{101\}$ facets - water molecules in the immediate vicinity of the nanocrystals are included. Oxygen atoms are shown in red, titanium atoms are shown in gray, hydrogen atoms are shown in white, and hydrogen bonds are shown as dashed, yellow lines. 

8 on anatase $\{001\}$, and 2 on anatase $\{101\}$. Experimentally OA has been observed to occur most commonly on $\{112\}$, occasionally on $\{001\}$, and rarely on $\{101\}$ surfaces of anatase [2]. We find that OA most frequently involves the surfaces that have the highest propensity to dissociate water. Our previous to a greater extent than the $\{101\}$ surface for water coverages up to $3.0 \mathrm{ML}$, which implies a higher hydroxyl coverage on the $\{112\}$ and $\{001\}$ facets than on $\{101\}$ [53]. The alignment of nanocrystals involves hydrogen bonds directed normal to their surfaces and this requires adsorbed hydroxyls. As shown in devoid of molecularly adsorbed water.

In the study where OA was first observed for nanocrystalline anatase, Penn and Banfield observe OA in deionized (DI) water as well as in acidic conditions [2]. The frequency of observed attachments in the most acidic solutions $(\mathrm{pH}$ observed in DI water. Our simulations indicate the aggregation mechanism in DI water. We also note that the OA mechanism observed in our simulations is consistent with that observed in liquid-phase HRTEM studies of OA for iron oxyhydroxide nanocrystals by Li et al. [19]. Thus, the OA mechanism observed in our simulations may be generic to aqueous oxide materials and indicates the important role played by water and hydrogen bonding in mediating crystal growth by OA.

\section{Acknowledgements}

This work was funded by the Department of Energy, Office of Basic Energy Sciences, Materials Science Division, grant number DE-FG02-07ER46414. This work used the resources of the Research Computing and Cyberinfrastructure of Information Technology Services at the Pennsylvania State University. 


\section{References}

[1] W. Ostwald, Studien über die bildung und umwandlung fester körper, Zeitschrift für physikalische Chemie 22 (1897) 289-330.

[2] R. L. Penn, J. F. Banfield, Morphology development and crystal growth in nanocrystalline aggregates under hydrothermal conditions: Insights from titania, Geochim. Cosmochim. Acta 63 (1999) 1549-1557.

[3] M. Niederberger, H. Cölfen, Oriented attachment and mesocrystals: Nonclassical crystallization mechanisms based on nanoparticle assembly, Phys. Chem. Chem. Phys. 8 (2006) 3271-3287.

[4] Q. Zhang, S.-J. Liu, S.-H. Yu, Recent advances in oriented attachment growth and synthesis of functional materials: Concept, evidence, mechanism, and future, J. Mater. Chem. 19 (2009) 191-207.

[5] J. Zhang, F. Huang, Z. Lin, Progress of nanocrystalline growth kinetics based on oriented attachment, Nanoscale 2 (2010) 18-34.

[6] Z. Tang, N. A. Kotov, One-dimensional assemblies of nanoparticles: Preparation, properties, and promise, Adv. Mater. 17 (2005) 951-962.

[7] K. S. Cho, D. V. Talapin, W. Gaschler, C. B. Murray, Designing PbSe nanowires and nanorings through oriented attachment of nanoparticles more options, J. Am. Chem. Soc. 127 (2005) 7140-7147.

[8] Y. Tian, H.-Y. Yang, K. Li, X. Jin, Monodispersed ultrathin GdF $_{3}$ nanowires: Oriented attachment, luminescence, and relaxivity for MRI contrast agents, J. Mater. Chem. 22 (2012) 22510-22516.

[9] J. H. Yu, J. Joo, H. M. Park, S. I. Baik, Y. W. Kim, S. C. Kim, T. Hyeon, Synthesis of quantum-sized cubic $\mathrm{ZnS}$ nanorods by the oriented attachment mechanism, J. Am. Chem. Soc. 127 (2005) 5662-5670.

[10] J. Sharma, T. Imae, Recent advances in fabrication of anisotropic metallic nanostructures, J. Nanoscience and Nanotechnology 9 (2009) 19-40. 
[18] H. G. Yang, H. C. Zeng, Self-construction of hollow $\mathrm{SnO}(2)$ octahedra based on two-dimensional aggregation of nanocrystallites, Angew. Chem. Int. Ed. Engl. 43 (2004) 5930-5933.

[19] D. Li, M. H. Nielsen, J. R. I. Lee, C. Frandsen, J. F. Banfield, J. J. De Yoreo,

[11] A. Halder, N. Ravishankar, Ultrafine single-crystalline gold nanowire arrays by oriented attachment, Adv. Mater. 19 (2007) 1854-1858.

[12] G. Zhang, W. Wang, X. Li, Solvothermal synthesis of V-VI binary and ternary hexagonal platelets: The oriented attachment mechanism, Cryst. Growth Des. 9 (2009) 145-150.

[13] C. Schliehe, B. H. Juarez, M. Pelletier, S. Jander, D. Greshnykh, M. Nagel, A. Meyer, S. Foerster, A. Kornowski, C. Klinke, H. Weller, Ultrathin PbS sheets by two-dimensional oriented attachment, Science 329 (2010) 550553.

[14] Z. Liu, H. Zhou, Y. S. Lim, J.-H. Song, L. Piao, S.-H. Kim, Synthesis of silver nanoplates by two-dimensional oriented attachment, Langmuir 28 (2012) 9244-9249.

[15] V. Yuwono, N. Burrows, J. Soltis, R. L. Penn, Oriented aggregation: Formation and transformation of mesocrystal intermediates revealed, J. Am. Chem. Soc. 132 (2010) 2163-2165.

[16] F. J. Heiligtag, M. D. Rossell, M. J. Suess, M. Niederberger, Template-free co-assembly of preformed $\mathrm{Au}$ and $\mathrm{TiO} 2$ nanoparticles into multicomponent 3D aerogels, J. Mater. Chem. 21 (2011) 16893-16899.

[17] D. Zitoun, N. Pinna, N. Frolet, C. Belin, Single crystal manganese oxide multipods by oriented attachment, J. Am. Chem. Soc. 127 (2005) 1503415035 .

Direction-specific interactions control crystal growth by oriented attachment, Science 336 (2012) 1014-1018. 
[20] H. L. Zhu, R. S. Averback, Sintering processes of two nanoparticles: A study by molecular-dynamics, Philos. Mag. Lett. 73 (1995) 27-33.

[21] J. S. Raut, R. B. Bhagat, K. A. Fichthorn, Sintering mechanisms of aluminum nanocrystals: A molecular-dynamics study, NanoStructured Mater. 10 (1998) 837-851.

[22] H. Zhang, J. F. Banfield, Aggregation, coarsening, and phase transformation in ZnS nanoparticles studied by molecular dynamics simulations, Nano Lett. 4 (4) (2004) 713-718.

[23] D. Spagnoli, J. F. Banfield, S. C. Parker, Free energy change of aggregation of nanoparticles, J. Phys. Chem. C 112 (38) (2008) 14731-14736.

[24] M. Alimohammadi, K. A. Fichthorn, Molecular dynamics simulation of the aggregation of titanium dioxide nanocrystals: Preferential alignment, Nano Lett. 9 (2009) 4198-203.

[25] R. Sathiyanarayanan, M. Alimohammadi, Y. Zhou, K. A. Fichthorn, Role of solvent in the shape-controlled synthesis of anisotropic colloidal nanostructures, J. Phys. Chem. C 115 (2011) 18983-18990.

[26] Y. Zhou, K. A. Fichthorn, Microscopic view of nucleation in the anataseto-rutile transformation, J. Phys. Chem. C 116 (2012) 8314-8321.

[27] M. Raju, A. C. T. van Duin, K. A. Fichthorn, Mechanisms of oriented attachment of TiO2 nanocrystals in vacuum and humid environments: Reactive molecular dynamics, Nano Lett. 14 (2014) 1836-1842.

[28] Y. Qin, K. A. Fichthorn, A molecular-dynamics simulation study of forces between colloidal nanoparticles in a Lennard-Jones liquid, J. Chem. Phys. 119 (2003) 9745-9755.

[29] K. A. Fichthorn, Y. Qin, Molecular dynamics simulation of colloidal nanoparticle forces, Ind. Eng. Chem. Res. 45 (2006) 5477-5481. 
[30] Y. Qin, K. A. Fichthorn, Solvophobicity at large and intermediate length scales: Size, shape, and solvent effects, Phys. Rev. E 74.

[31] Y. Qin, K. A. Fichthorn, Molecular dynamics simulation of the forces between colloidal nanoparticles in n-decane solvent, J. Chem. Phys. 127 (2007) 144911.

[32] K. A. Fichthorn, Y. Qin, Molecular dynamics simulation of the forces between colloidal nanoparticles in Lennard-Jones and n-decane solvent, Granul. Matter 10 (2008) 105-111.

[33] M. Lazzeri, A. Vittadini, A. Selloni, Structure and energetics of stoichiometric TiO2 anatase surfaces, Phys. Rev. B 63 (2001) 1-9.

[34] A. S. Barnard, P. Zapol, Predicting the energetics, phase stability, and morphology evolution of faceted and spherical anatase nanocrystals, J. Phys. Chem. C 108 (2004) 18435-18440.

[35] M. Matsui, M. Akaogi, Molecular dynamics simulation of the structural and physical properties of the four polymorphs of TiO2, Mol. Simul. 6 (1991) 239-244.

[36] J. G. Traylor, H. G. Smith, R. M. Nicklow, M. K. Wilkinson, Lattice dynamics of rutile, Phys. Rev. B 3 (1971) 3457-3472.

[37] D. R. Collins, W. Smith, Research Report DL-TR-96-001, Council for the Central Laboratory of Research Councils, Daresbury, UK, 1996.

[38] R. D. Eithiraj, K. R. Geethalakshmi, Suitability of amorphous TiO2 nanoparticles as a photoelectrode in dye sensitized solar cells: A DFTTDDFT study, Chem. Phys. Lett. 585 (2013) 138-142.

[39] J. Houska, S. Mraz, J. M. Schneider, Experimental and molecular dynamics study of the growth of crystalline TiO2, J. Appl. Phys. 112 (2012) 073527.

[40] S. Salameh, J. Schneider, J. Laube, A. Alessandrini, P. Facci, J. W. Seo, L. C. Ciacchi, L. Maedler, Adhesion mechanisms of the contact interface 
of TiO2 nanoparticles in films and aggregates, Langmuir 28 (2012) 1145711464 .

[41] P. M. Oliver, G. W. Watson, E. T. Kelsey, S. C. Parker, Atomistic simulation of the surface structure of the $\mathrm{TiO} 2$ polymorphs rutile and anatase, J. Mater. Chem. 7 (1997) 563-568.

[42] A. V. Bandura, J. D. Kubicki, Derivation of force field parameters for TiO2H2O systems from ab initio calculations, J. Phys. Chem. B 107 (2003) 11072-11081.

[43] S. Hamad, C. R. A. Catlow, S. M. Woodley, S. Lago, J. A. Mejias, Structure and stability of small TiO2 nanoparticles, J. Phys. Chem. B 109 (2005) $15741-15748$.

[44] N. J. English, D. C. Sorescua, J. K. Johnson, Effects of an external electromagnetic field on rutile TiO2: A molecular dynamics study, J. Phys. Chem. Solids 67 (2006) 1399-1409.

[45] N. A. Dubrovinskaia, L. S. Dubrovinsky, R. Ahuja, V. B. Prokopenko, V. Dmitriev, H.-P. Weber, J. M. Osorio-Guillen, B. Johansson, Experimental and theoretical identification of a new high-pressure TiO2 polymorph, Phys. Rev. Lett. 87 (2001) 275501.

[46] V. Swamy, J. D. Gale, Transferable variable-charge interatomic potential for atomistic simulation of titanium oxides, Phys. Rev. B 62 (2000) 54065412 .

[47] V. Swamy, J. D. Gale, L. S. Dubrovinsky, Atomistic simulation of the crystal structures and bulk moduli of $\mathrm{TiO} 2$ polymorphs, J. Phys. Chem. Solids 62 (2001) 887-895.

[48] B. S. Thomas, N. A. Marks, B. D. Begg, Empirical variable-charge models for titanium oxides: A study in transferability, Phys. Rev. B 69 (2004) 144122 . 
[49] Z. Tang, N. Kotov, M. Giersig, Spontaneous organization of single CdTe nanoparticles into luminescent nanowires, Science 297 (2002) 237-240.

[50] D. Talapin, E. Shevchenko, C. Murray, Dipole-dipole interactions in nanoparticle superlattices, Nano Lett. 7 (2007) 1213-1219.

[51] W. Smith, T. R. Forester, DL-POLY 2.0: A general-purpose parallel molecular dynamics simulation package, J. Mol. Graph. 14 (1996) 136-141.

[52] S.-Y. Kim, N. Kumar, P. Persson, J. Sofo, A. C. T. van Duin, J. D. Kubicki, Development of a ReaxFF reactive force field for titanium dioxide/water systems, Langmuir 29 (2013) 7838-7846.

[53] M. Raju, S.-Y. Kim, A. C. T. van Duin, K. A. Fichthorn, ReaxFF reactive force field study of the dissociation of water on titania surfaces, J. Phys. Chem. C 117 (2013) 10558-10572. 